\title{
Modelling evaporation processes of cement-bentonite mixtures
}

\author{
Vincenzo Sergio Vespo ${ }^{1}$, Gabriele Della Vecchia ${ }^{2}$, and Guido Musso ${ }^{1, *}$ \\ ${ }^{1}$ Politecnico di Torino, DISEG, Corso Duca degli Abruzzi 24, 10129 Torino, Italy \\ ${ }^{2}$ Politecnico di Milano, DICA, Piazza Leonardo da Vinci 32, 20133 Milano, Italy
}

\begin{abstract}
Upon drying, matter and energy are exchanged between the atmosphere and porous media through evaporation, which is a coupled process that involves the simultaneous transport of liquid water, water vapour and heat. At shallow depths, evaporation controls the water content and suction of both natural soils and earthworks, affecting their hydraulic response. This impact is particularly relevant when the earthworks are aimed at the containment of aqueous or non-aqueous pollutants, as in the case of cement bentonite cut-off walls. A coupled model for the transport of liquid water, water vapour and heat through cement bentonite mixtures upon evaporation was formulated. The model considers flow of water driven by both total suction and temperature gradients. Model predictions were compared to experimental results obtained in the laboratory on samples having different sizes and imposed boundary conditions. A good agreement between predicted and measured volumetric water contents was obtained, once defined a suitable dependency of the relative permeability of the mixture on degree of saturation. The results suggest that the proposed formulation correctly accounts for the underlying physical processes, and that it might be used to model the real scale behaviour of cut-off walls.
\end{abstract}

\section{Introduction}

The contemporaneous movement of heat, water vapour, and liquid water plays an important role in many geotechnical and geo-environmental applications. In the context of waste disposal, the prediction of the volume of leachate percolating from landfills requires the analysis of the heat and of the water mass exchanged through the landfill cover (see e.g. [1]). In the context of nuclear waste storage, the radioactive decay may generate high temperatures and temperature gradients, which are expected to cause evaporation of water, soil desaturation, and consequent migration and condensation of water vapour in cooler areas (e.g. [2]). Simultaneous fluxes of heat and liquid and vapour water are inherently relevant in the exploitation of geothermal energy and in the remediation of polluted lands through soil venting technique [3,4].

This article focuses on the so called "slurry trench cut-off walls", widely used for the containment of both aqueous and non-aqueous pollutants. The construction process is normally based on a series of trenches excavated and filled up with a slurry of materials having low hydraulic conductivity. Cement-bentonite mixtures are normally used. After a curing period, the mixture hardens and acquires a relatively high strength and low hydraulic conductivity (uniaxial strength higher than $200 \mathrm{kPa}$ and hydraulic conductivity $\mathrm{K}_{\mathrm{w}}<10^{-8} \mathrm{~m} / \mathrm{s}$ ) [5-7].

In many operating conditions the shallower portions of the cut-off walls are expected to lay above the water table and then to interact with the atmosphere, through evaporation and rain infiltration. The permeability of the walls is strongly influenced by the water saturation and desaturation might have undesired effects, especially when the barrier is aimed at the containment of nonaqueous pollutants that rest above the water table (LNAPL). Preliminary works suggest that dryingwetting cycles might cause cracks in the barrier [8-9].

This work focuses on the study of the evaporation processes occurring in cement-bentonite mixtures. A set of differential equations, defining the mass balance of liquid water and vapour and the heat balance, is proposed. The parameters of these differential equations, describing the water retention and the transport laws were calibrated upon previous characterization [10] and laboratory data from evaporation experiments [11].

\section{Formulation of the evaporation model}

The thermo-hydraulic model accounts for the mass balance of water in its liquid and gaseous states and for the balance of energy (heat). The Darcy's law, extended to unsaturated conditions, describes flow of liquid water due to water pressure gradients. The Fick's law describes the flux of water vapour due to its diffusion through the air phase and driven by the concentration gradient of water molecules in air. The volume of liquid water retained within the porous medium is related to suction through the water retention curve, while the concentration of water molecules in air is related to suction through the Kelvin's psychrometric law. Energy balance considers the transport of heat by conduction and transfer of latent heat by diffusion of water vapour.

* Corresponding author: guido.musso@polito.it 


\subsection{Mass balance equation for water as a chemical species}

Two-phase flow problems are often solved by considering two mass balance equations, one for each fluid phase. Molecules of water and dissolved air compose the liquid phase. Molecules of dry air and water vapour compose the gas phase. Through evaporation, molecules of water are exchanged between the liquid and the gas phase. The exchange is modelled by introducing mass transfer terms $f_{a \rightarrow b}$ in both equations, where $a$ is phase that is modelled and $b$ is the other one. The number of water molecules leaving the liquid phase is equal to the number of water molecules entering the gas phase and the mass transfer terms are equal in magnitude and opposite in sign: $f_{l \rightarrow g}=-f_{g \rightarrow l}$.

The mass balance equations can be as well expressed in terms of chemical species. The flux and the mass of every chemical species in each phase are summed, with the advantage that through summation the transfer terms cancel out [12]. In the following, the mass balance of air is neglected, while the mass balance of water assumes the form:

$$
\begin{aligned}
& \frac{\partial}{\partial t}\left[\phi\left(S_{l} c_{l}^{w}+S_{g} c_{g}^{w}\right)\right]+\nabla \cdot\left(c_{l}^{w} \mathbf{q}_{l}+c_{g}^{w} \mathbf{q}_{g}\right)+ \\
& +\nabla \cdot \phi\left(S_{l} \mathbf{J}_{h l}^{w}+S_{g} \mathbf{J}_{h g}^{w}\right)=S_{l} \phi \rho_{l} \Gamma_{l}^{w}+S_{g} \phi \rho_{g} \Gamma_{g}^{w}
\end{aligned}
$$

where $\phi$ is porosity; $S_{l}$ and $S_{g}$ are the liquid and gas degree of saturation $\left(S_{l}=1-S_{g}\right) ; c_{l}^{w}$ and $c_{g}^{w}$ are the water mass concentration in the liquid and gas phase; $\mathbf{q}_{l}$ and $\mathbf{q}_{g}$ are the advective fluxes of the liquid and gas phase; $\mathbf{J}_{h l}^{w}$ and $\mathbf{J}_{h g}^{w}$ represent the sum of diffusive and dispersive fluxes of water in liquid and gas phase; $\rho_{l}$ and $\rho_{g}$ are the mass density of the liquid and of the gas phase; $\Gamma_{l}^{w}$ and $\Gamma_{g}^{w}$ are the generation rate of water mass per unit mass of liquid and gas phase.

Neglecting source terms, dispersive flux of water vapour, the air dissolved in the liquid (from which $c_{l}^{w}=\rho_{l}$ and $\left.\mathbf{J}_{h l}^{w}=0\right)$, and under the assumption that air pressure is constant over time and space (from which $\mathbf{v}_{g}=0$ ), equation (1) becomes:

$$
\frac{\partial}{\partial t}\left[\phi\left(S_{l} \rho_{l}+S_{g} c_{g}^{w}\right)\right]+\nabla \cdot\left(\rho_{l} \mathbf{q}_{l}\right)+\nabla \cdot \phi\left(S_{g} \mathbf{J}_{g}^{w}\right)=0
$$

\subsubsection{Flow equations for liquid and vapour water}

The specific discharge of liquid water in an unsaturated material can be described using Darcy's law (3), while diffusive flux of water vapour in an unsaturated material can be described using Fick's law (4):

$$
\begin{gathered}
\mathbf{q}_{l}=-\frac{\mathbf{K}_{w}\left(S_{l}\right)}{\rho_{l} g} \nabla\left(u_{w}+\rho_{l} g z\right) \\
\mathbf{J}_{g}^{w}=-\mathbf{D} \nabla c_{g}^{w}
\end{gathered}
$$

where: $\mathbf{K}_{w}$ is the unsaturated hydraulic conductivity tensor, $g$ is gravitational acceleration, $u_{w}$ is water pressure, $z$ is the elevation head, and $\mathbf{D}$ is the water vapour diffusion tensor for the porous medium.

\subsubsection{Relationships for water retention, vapour concentration and water flow}

The link between matric suction and the quantity of water stored in the pores is provided by water retention curve, here modelled through the van Genuchten equation [13]:

$$
S_{r}^{e f f}=\left[1+(\alpha s)^{n}\right]^{-m}
$$

where $\alpha, n$ and $m$ are model parameters of medium and $s=u_{a}-u_{w}$ is matric suction, $u_{a}$ being the air pressure. $S_{r}^{e f f}$ is the effective water saturation:

$$
S_{r}^{e f f}=\frac{\left(S_{l}-S_{r}^{r e s}\right)}{\left(1-S_{r}^{r e s}\right)}
$$

with $S_{r}^{\text {res }}$ is the irreducible degree of water saturation.

The psychrometric law provides the link between relative humidity $h_{r}$ and total suction $\Psi$ [14]:

$$
h_{r}=e^{\frac{-\Psi M^{w}}{\rho_{l} R T}}
$$

where $M^{w}$ is molecular weight of water, $R$ is universal gas constant and $T$ is temperature. This allows to evaluate the concentration of water in the gas phase according to:

$$
c_{g}^{w}=c_{g, s a t}^{w} \cdot h_{r}
$$

where $c_{g, s a t}^{w}$ is the saturated concentration of water vapour that in turn is related to saturated vapour pressure of water vapour $p_{v, \text { sat }}$ by the ideal gas law:

$$
c_{g, s a t}^{w}=\frac{p_{v, s a t} \cdot M^{w}}{R T}
$$

The saturated vapour pressure of water vapour can be finally calculated as follows [15]:

$$
p_{v, \text { sat }}=6.108 \cdot 10^{-4} \cdot 10^{\frac{7.5 \cdot(T-273.15)}{T}}
$$

The water vapour diffusion tensor is set equal to

$$
\mathbf{D}=D_{v} \cdot \boldsymbol{\tau}
$$

where $D_{v}$ is the diffusivity coefficient for the vapour in free air and $\boldsymbol{\tau}$ is a tensor which accounts for the tortuosity of path of the molecules of water vapour through the porous medium.

The diffusivity coefficient for the vapour in free air can be calculated as follows [16] 


$$
D_{v}=0.229 \cdot 10^{-4}\left(1+\frac{T}{273}\right)^{1.75}
$$

The hydraulic conductivity in unsaturated conditions was modelled as:

$$
\mathbf{K}_{w}=\mathbf{K}_{w}^{s a t} \cdot k_{r}
$$

where $\mathbf{K}_{w}^{\text {sat }}$ is the hydraulic conductivity for $S_{l}=1$ and $k_{r}$ is the relative permeability. In order to reproduce as well as the transient behaviour of the cementbentonite mixture studied through laboratory tests, $k_{r}$ was defined through a modified van Genuchten expression:

$$
k_{r}=\left(S_{r}^{e f f}\right)^{1 / 2}\left\{1-\left[1-\left(S_{r}^{e f f}\right)^{1 / m}\right]^{m}\right\}^{\beta}
$$

In the original formulation, $\beta=2$ however, in this work, a better result was obtained by taking $\beta=8$. A similar approach was adopted in [17], which used $k_{r}=S_{l}^{12}$ to model the relative permeability of clay during a heating test.

\subsection{Energy balance}

The governing equation for the balance of energy in a variably saturated porous medium is given by the energy conservation equation [15]:

$$
c_{v} \frac{\partial T}{\partial t}=-\nabla \cdot\left(\mathbf{q}_{h}\right)-\mathbf{Q}
$$

where $c_{v}$ is volumetric specific heat of the porous medium, $\mathbf{q}_{h}$ is the total heat flux, and $\mathbf{Q}$ accounts for energy sources and sinks.

\subsubsection{Heat flux}

The total heat flux is defined as the sum of the conduction of sensible heat as described by Fourier's law and latent heat by vapour flow:

$$
\mathbf{q}_{h}=-k_{c} \nabla T+L_{v} \mathbf{J}_{g}^{w}
$$

where $k_{c}$ is thermal conductivity of the porous medium, and $L_{v}$ is latent heat of vaporization for water. The sensible heat by convection of liquid and vapour water are not included because negligible as evidenced by $[18]$.

The latent heat of vaporization for water can be calculated as follows [15]:

$$
L_{v}=10^{-6} \cdot\left[2.5-2.37 \cdot 10^{-3} \cdot(T-273.15)\right]
$$

\subsubsection{Energy transport parameters for multiphase materials}

The volumetric specific heat of the mixture is determined using the method described by de Vries [19]:

$$
c_{v}=c_{v, s} \cdot \theta_{s}+c_{v, w} \cdot \theta_{w}+c_{v, a} \cdot \theta_{a}
$$

where: $c_{v, s}, c_{v, w}$, and $c_{v, a}$ are volumetric specific heat capacity of the soil solids, liquid water phase, and air phase (which can be assumed to be negligible), respectively; $\theta_{s}, \theta_{w}$, and $\theta_{a}$ are volumetric fractions of the soil solids, water, and air, respectively.

The thermal conductivity $k_{c}$ of the cement-bentonite mixture is also a function of the water content of the material. The following equation (see [20]) was used:

$$
k_{c}=\left(\theta_{s} \sqrt{k_{c, s}}+\theta_{w} \sqrt{k_{c, w}}+\theta_{a} \sqrt{k_{c, a}}\right)^{2}
$$

where: $k_{c, s}, k_{c, w}$, and $k_{c, a}$ are thermal conductivity of the soil solids, liquid water phase, and air phase, respectively.

\subsection{Coupled equations for the water and energy balance}

Under the assumption that osmotic suction is constant over time and space, $s$ can be replaced with $\Psi$. A form of the mass balance equation of water, suitable for modelling the coupled processes involved in the evaporation, is then obtained introducing the flow laws (eq. (3) and (4)), the water retention (5) and the psychometric law (7) into eq. (2). Considering the compressibility of liquid water negligible, it follows:

$$
\begin{gathered}
\frac{\partial \Psi}{\partial t}\left[S_{l} \rho_{l} m_{e}-\rho_{l} C_{w}+c_{g}^{w}\left(1-S_{l}\right) m_{e}+c_{g}^{w} C_{w}-\phi\left(1-S_{l}\right)\left(\frac{c_{g}^{w} M_{w}}{\rho_{l} R T}\right)\right]+ \\
+\nabla \cdot\left\{\left[\frac{\mathbf{K}_{w}^{s a t} \cdot k_{r}}{g} \nabla\left(\Psi-\rho_{l} g z\right)-\phi D_{v} \tau\left(1-S_{l}\right) \frac{c_{g}^{w} M_{w}}{\rho_{l} R T}\left(\frac{\Psi}{T} \nabla T-\nabla \Psi\right)\right]=\right. \\
=-\frac{\partial T}{\partial t}\left[\phi\left(1-S_{l}\right) \frac{c_{g}^{w} M_{w} \Psi}{\rho_{l} R T^{2}}\right]
\end{gathered}
$$

where: $C_{w}$ is water capacity:

$$
C_{w}=-\phi \frac{\partial S_{l}}{\partial \Psi}
$$

and

$$
m_{e}=\frac{\partial \phi}{\partial \Psi}
$$

Neglecting sources and sinks of energy, the following energy balance equation is obtained by introducing eq. (16) into eq. (15):

$$
\begin{gathered}
c_{v} \frac{\partial T}{\partial t}+\nabla \cdot\left[\left(-k_{c}-L_{v} D^{w} \tau \frac{c_{g}^{w} M^{w} \Psi}{\rho_{l} R T^{2}}\right) \nabla T+\right. \\
\left.+\left(L_{v} D^{w} \tau \frac{c_{g}^{w} M^{w}}{\rho_{l} R T}\right) \nabla \Psi\right]=0
\end{gathered}
$$

Eq. (20) and (23) provide the system of coupled Partial Differential Equations describing the evaporation process, in terms of total suction and temperature. 


\section{Evaporation experiments}

\subsection{Materials and sample preparation}

The cement-bentonite mixture, used for the experimental tests carried out at Politecnico di Torino [10, 11], was obtained mixing a commercial cement (CEM I $32.5 \mathrm{~N}$, Portland 325) with a commercial sodium-calcium bentonite provided by Laviosa Chimica Mineraria (Livorno, Italy). According to preliminary characterization, its liquid limit is $535 \%$, the plastic limit is $75 \%$ and the specific gravity is 2.95 .

The bentonite powder was first mixed with tap water in a mixer until a homogeneous plastic paste was obtained. The imposed mass ratio between water and bentonite was 18:1. The paste was then covered with an impermeable sheet and left to rest for 24 hours to ensure full hydration of the bentonite and homogenization of the water content. The cement powder was finally added to the hydrated bentonite and mixed again. The mass ratio between cement and bentonite was $4: 1$.

The cement-bentonite mixture was carefully poured into plastic moulds placed at the bottom of large boxes. After pouring, the boxes were filled with tap water, and the samples were left to cure under water for 28 and 60 days. The specific weight of the cured samples was determined to be $G_{s}=2.68$. Further details on the curing and preparation processes can be found in $[10,11]$.

\subsection{Tests}

Three samples were used for the evaporation tests (Table 1).

Table 1. Features of samples.

\begin{tabular}{|c|c|c|c|c|}
\hline & $\begin{array}{c}\text { Height } \\
{[\mathrm{cm}]}\end{array}$ & $\begin{array}{c}\text { Diameter } \\
{[\mathrm{cm}]}\end{array}$ & $\begin{array}{c}\text { Curing } \\
{[\text { day }]}\end{array}$ & $\begin{array}{c}\text { Test time } \\
{[\text { day }]}\end{array}$ \\
\hline A & 4 & 11 & 28 & 10 \\
\hline B & 4 & 11 & 60 & 8 \\
\hline C & 4 & 19 & 28 & 6 \\
\hline
\end{tabular}

The drying process was induced by exposure of samples to the laboratory environment (temperature equal to $294 \mathrm{~K}$ and relative humidity of $38 \%$ ). The variation of total mass, and therefore the loss of water mass through evaporation, was evaluated by progressively recording the weight of the specimens as the drying process progressed. The mass of solid was determined by drying the specimens in oven at $105^{\circ} \mathrm{C}$ for 24 hours after the end of the tests.

Below a water content of about $100 \%$, the behaviour of the material was extremely fragile and it was not possible to continue the tests due to the crumbling of specimens when moved to the balance.

\section{Numerical model}

The numerical solution of the equations (20) and (23) was obtained using COMSOL Multiphysics ${ }^{\circledR}$, a finite element software that allows solving coupled multiphysics problems. A 2D axisymmetric geometry with a mesh of 670 triangular elements was used.

Boundary conditions for flow equation and energy balance have been chosen to reproduce laboratory conditions. Regarding the mass balance equation for water, a constant value of total suction (related to relative humidity) was imposed at the top and lateral boundaries ( $\Psi=131.5 \mathrm{MPa}$ ), while the bottom boundary has been set to be impervious (i.e. no-flow condition). As for the energy balance, the constant temperature of laboratory $(T=294 \mathrm{~K})$ was imposed on all boundaries.

An initial void ratio $e_{0}=8.31$, initial temperature $T_{0}=294 \mathrm{~K}$, initial total suction $\Psi_{0}=10 \mathrm{kPa}$ were employed.

The volumetric specific heat and the thermal conductivity of the soil solids were taken as the average values between the cement paste and bentonite [21, 22], the volumetric specific heat of liquid water recommended by [19] was used, and the thermal conductivity of liquid water phase and air phase recommend by [20] were used. Values of the model parameters for mixture, used in the simulations, are shown in Table 2.

Table 2. Volumetric specific heat and thermal conductivity used in numerical models.

\begin{tabular}{|c|c|c|c|c|}
\hline $\begin{array}{c}c_{v, s} \\
{\left[\mathrm{~J} /\left(\mathrm{m}^{3} \cdot \mathrm{K}\right)\right]}\end{array}$ & $\begin{array}{c}c_{v, w} \\
{\left[\mathrm{~J} /\left(\mathrm{m}^{3} \cdot \mathrm{K}\right)\right]}\end{array}$ & $\begin{array}{c}k_{c, s} \\
{[\mathrm{~W} /(\mathrm{m} \cdot \mathrm{K})]}\end{array}$ & $\begin{array}{c}k_{c, w} \\
{[\mathrm{~W} /(\mathrm{m} \cdot \mathrm{K})]}\end{array}$ & $\begin{array}{c}k_{c, a} \\
{[\mathrm{~W} /(\mathrm{m} \cdot \mathrm{K})]}\end{array}$ \\
\hline $2.968 \cdot 10^{6}$ & $4.186 \cdot 10^{6}$ & 1.345 & 0.6 & 0.024 \\
\hline
\end{tabular}

The water retention, the hydraulic conductivity, and the relationship between void ratio and suction were obtained through independent experiments [10]. The following power law was used to fit the experimental relationship between void ratio and suction:

$$
e=e_{0}\left(\frac{\Psi}{\Psi_{0}}\right)^{-0.043}
$$

Table 3 provides the values of model parameters used in the simulations.

Table 3. Water retention and hydraulic conductivity used in numerical models.

\begin{tabular}{|c|c|c|c|c|}
\hline $\begin{array}{c}\alpha \\
{\left[\mathrm{MPa}^{-1}\right]}\end{array}$ & $\begin{array}{c}n \\
{[-]}\end{array}$ & $\begin{array}{c}m \\
{[-]}\end{array}$ & $\begin{array}{c}S_{r}^{\text {res }} \\
{[-]}\end{array}$ & $\begin{array}{c}K_{w} \\
{[\mathrm{~m} / \mathrm{s}]}\end{array}$ \\
\hline 4.436 & 1.618 & 0.382 & 0.02 & $10^{-8}$ \\
\hline
\end{tabular}

\section{Results and discussion}

The cement-bentonite mixture was assumed to be isotropic and the value of the scalar tortuosity $\tau$ for each sample was determined through back analysis of the 
experimental tests $[10,11]$. The results are summarized in Table 4.

Table 4. Tortuosity values.

\begin{tabular}{|c|c|c|}
\hline Specimen A & Specimen B & Specimen C \\
\hline 0.08 & 0.12 & 0.05 \\
\hline
\end{tabular}

Although in theory tortuosity should vary with water saturation and porosity, its determination is difficult and assuming a constant value based on back analysis is widely used in literature [23]. The higher value of $\tau$ for specimen $\mathrm{B}$ with respect specimens $\mathrm{A}$ and $\mathrm{C}$ might be related to larger curing time.

Fig. 1 shows the numerical predictions of gravimetric water content evolution over time for each specimen, together with experimental results. The good match between the simulations and the experiments confirms the validity of the evaporation model with the modified van Genuchten expression of eq. (14). Model predictions are very sensitive to parameter $\beta$ : analysis with $\beta=2$ (as suggested in the original model) overestimated the decrease rate of water content, while for $\beta>8$ the computational effort increased without any benefit in terms of prediction quality.

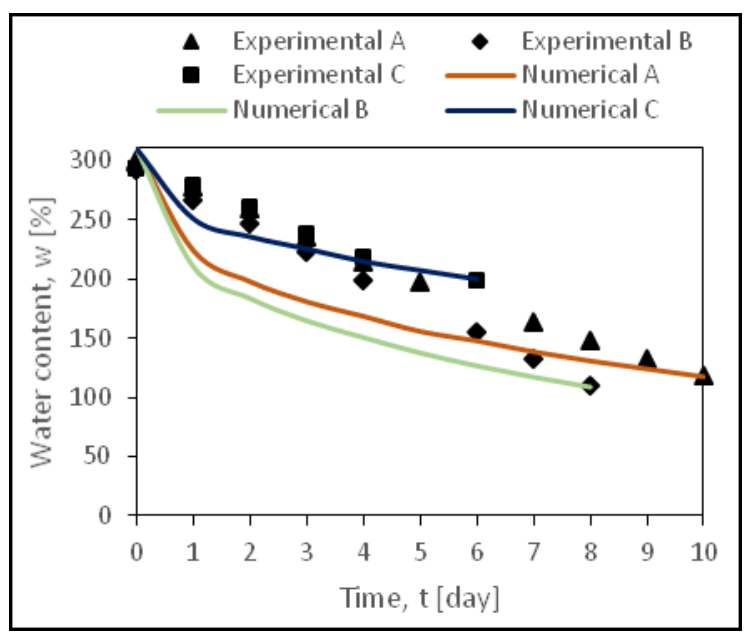

Fig. 1. Observed and predicted evolution of water content with time.

Fig. 2 shows water content profiles computed at four different times for specimen A along the symmetry axis. These profiles illustrate the progressive desaturation of the mixture from its top. The water content decreases over time to values near residual saturation at a depth that grows with time. Saturation at the top achieves dry conditions and the bottom of the dry zone represents the location of the evaporation front. Below the evaporation front, the water content continuously increases with depth.

Coupling between heat and hydraulic processes causes changes of temperature within the sample. The temperature profiles in Fig. 3 show the movement from top to bottom of the evaporation front, which is located at the depth where the lowest temperature is recorded. The evaporation front in fact acts as a heat sink.

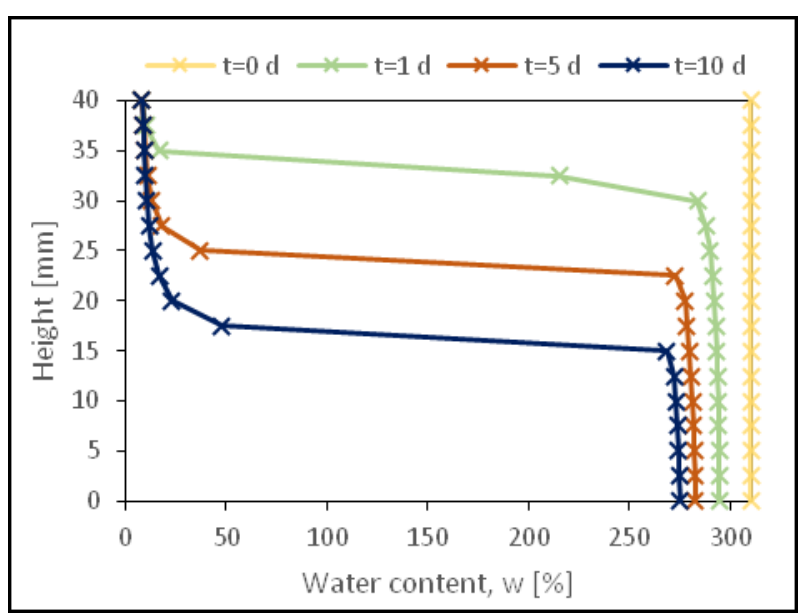

Fig. 2. Computed profiles of water content for specimen A.

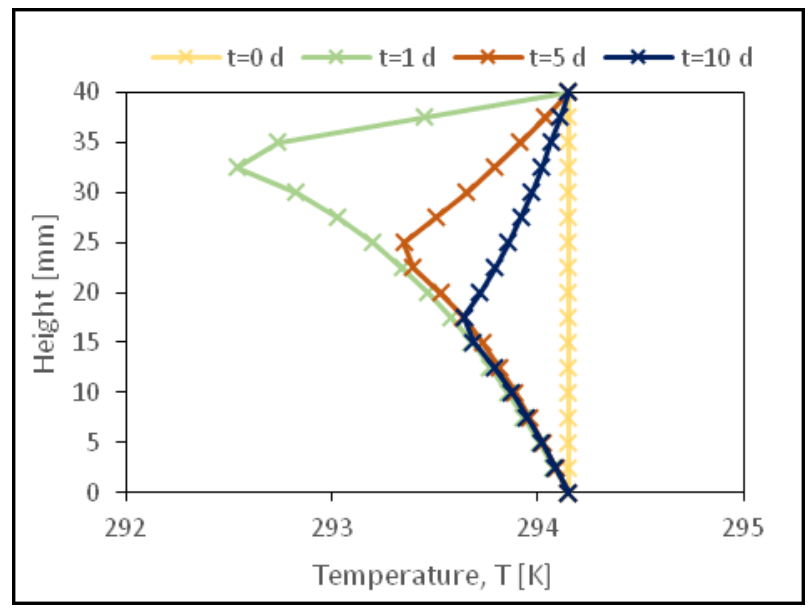

Fig. 3. Computed profiles of temperature for specimen A.

Fig. 4 and 5 display the profiles of liquid water and water vapour fluxes. A continuous upward liquid water flux occurs below the drying front. Liquid water is moved, because of capillarity, from the deeper layers toward the drying front, where it is converted into water vapour. Above the drying front, the upward water vapour flux is the dominant flux component, and in this region water is further transported as water vapour, mainly by the suction gradient, toward the surface.

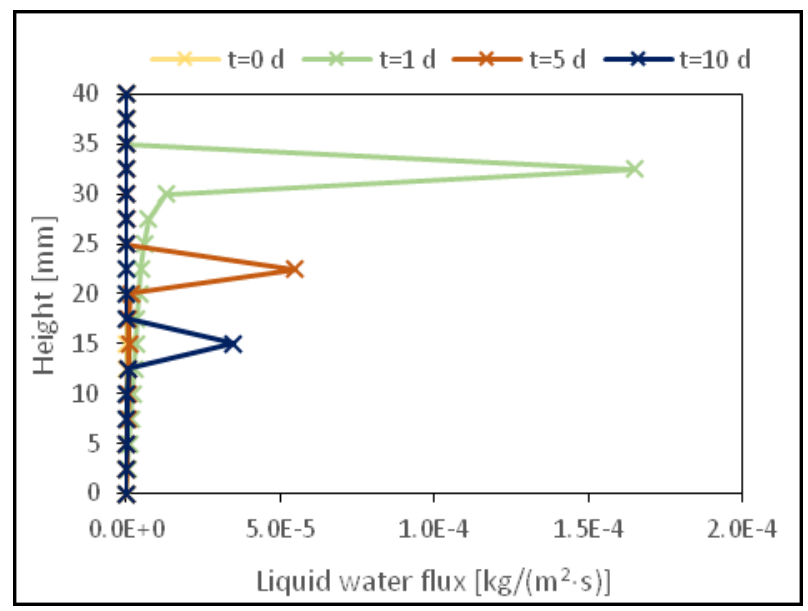

Fig. 4. Computed profiles of liquid water flux for specimen A. 


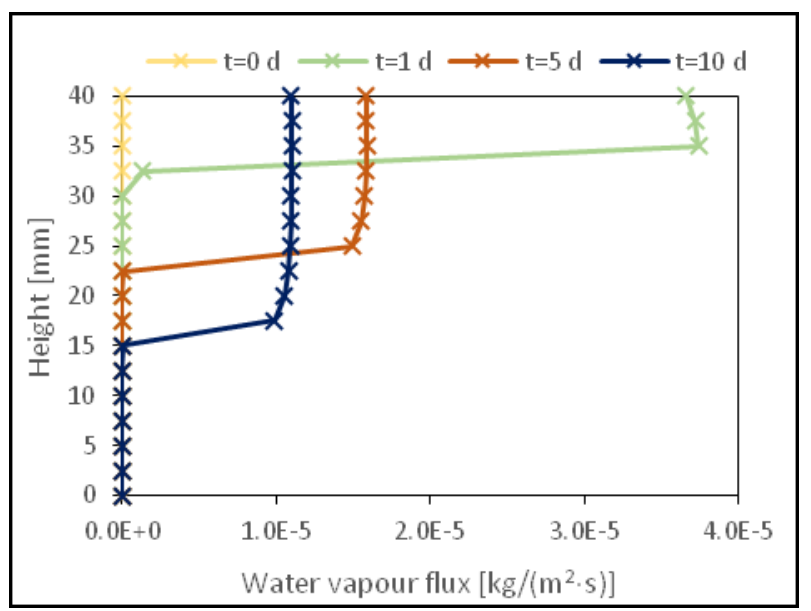

Fig. 5. Computed profiles of water vapour flux for specimen A.

\section{Concluding remarks}

Some preliminary results of a theoretical and numerical investigation on the processes governing water evaporation from cement-bentonite mixtures were presented. The general formulation of the evaporation problem, accounting for the coupled transport of water mass and heat through a deformable porous material, was described first.

While most model parameters were obtained through independent experiments or from theoretical expressions, the relative permeability function and the tortuosity of the hydraulic paths were determined through back analysis of drying tests. The relative permeability function derived through back analysis suggests a marked drop of hydraulic conductivity as the degree of liquid saturation decreases. On the other hand, the relatively high values of the coefficient describing the tortuosity of the vapour path suggest that the vapour diffusion is a quite effective transport mechanism for this material. If confirmed by ongoing bench-scale experiments and by field scale simulation, this aspect would support the requirement for impermeable cover systems to be placed on top of the cut-off walls, in order to prevent desiccation processes which might lead to loss of strength and integrity in the shallower layers of this barriers.

\section{References}

1. M. V. Khire, C. H. Benson, P. J. Bosscher, J. Geotech. Geoenviron. Eng., 123, 744-754 (1997)

2. N. F. Spycher, E. L. Sonnenthal, J. A. Apps,. J. Contam. Hydrol., 62-63, 653-673 (2003)

3. C. R. Faust, J. W. Mercer, Water Resour. Res., 15 (1), 23-30 (1979)

4. R. W. Falta, K. Pruess, I. Javandel, P. A. Witherspoon, Water Resour. Res., 28 (2), 433-449 (1992)

5. S. A. Jefferis, in Proceedings, Tenth International Conference on Soil Mechanics and Foundation
Engineering, Stockholm, Sweden, Vol. 1, 435-440 (1981)

6. K. Soga, K. Joshi, J. C. Evans., in Coupled Phenomena in Environmental Geotechnics. Proc. Int. Symp., ISSMGE TC 315, M. Manassero et al. eds, Taylor and Francis, 149-165, (2013).

7. G. Scelsi, G. Della Vecchia, C. di Prisco, G. Musso, G. Sanetti., in National Conference of the Researchers of Geotechnical Engineering. Springer, Cham, 555-564 (2019)

8. A. C. D. Royal, A. W. Opukumo, C. S. Qadr, L. M. Perkins, M. A. Walenna, Geotech Geol Eng, 36, 835-853 (2018)

9. V. S. Vespo, G. Musso, G. Guida, G. Della Vecchia, IAGIG 2020 (to be published)

10. P. Trischitta, R. M. Cosentini, G. Della Vecchia, G. Musso, e-unsat 2020 (to be published)

11. G. Musso, A Zibisco, R. M. Cosentini, P. Trischitta, G. Della Vecchia, e-unsat 2020 (to be published)

12. J. Bear, A. H. D. Cheng, Modeling Groundwater Flow and Contaminant Transport (Springer Science \& Business Media, Netherlands, 2010)

13. M. T. van Genuchten, Soil Science Society of America Journal, 44 (5), 892-898 (1980)

14. N. E. Edlefsen, A. B. C. Anderson, Thermodynamics of soil moisture. Hilgardia, 15 (2), 31-298 (1943)

15. J. A. Tindall, J. R. Kunkel, D. E. Anderson, Unsaturated Zone Hydrology for Scientists and Engineers (Prentice Hall, New Jersey, 1998)

16. B. A. Kimball, R. D. Jackson, R. J. Reginato, F. S. Nakayama, S. B. Idso, Soil Science Society of America Proceedings, 40 (1), 18-25 (1976)

17. S. Olivella, A. Gens, Transport in Porous Media, 40, 219-241 (2000)

18. P. C. D. Milly, Water resources Research, 20 (8), 1075-1085 (1984)

19. D. A. de Vries, in Physics of plant environment (edited by W. R. Van Wijk, Netherlands), 210-235 (1963)

20. P. Cosenza, R. Guérin, A. Tabbagh, European Journal of Soil Science, 54, 581-587 (2003)

21. S. Yoon, D. E. Macphee, M. S. Imbabi, Thermochimica Acta, 588, 1-10 (2014)

22. S. Knutsson, On the thermal conductivity and thermal diffusivity of highly compacted bentonite (Luleå, Sweden, 1983)

23. M. Gran, J. Carrera, S. Olivella, M. W. Saaltink, Hydrol. Earth Syst. Sci. Discuss., 8, 529-554 (2011) 